\title{
Commemorating the 50th Anniversary of the Laser
}

\author{
Gregory J. Quarles \\ BE Meyers \& Co. \\ 14540 NE 91st Street \\ Redmond, Washington 98052 \\ Yehoshua Kalisky \\ Nuclear Research Center Negev \\ Chemistry Division \\ PO Box 9001 \\ Beer-Sheva, 84190 Israel
}

The roots of the basic concepts of laser theory were first established by Einstein in 1917, and later the necessary conditions for maser and laser operation, as well as the first concepts of quantum electronics and laser optics, were developed. This theoretical foundation was followed by the experimental demonstration of the first ruby laser operation by Theodore Maiman in May 1960. This first laser operation was a significant technological breakthrough with a myriad of implications spanning all fields of science and technology. Maiman's laser demonstration triggered an accelerated growth in research and development of other types of lasers and their applications, such as gas, liquid (dye lasers), and other novel solid-state lasers, materials, optical elements, and devices, with simultaneous rapid developments in laser physics, quantum optics, and nonlinear optics. This modest special section of Optical Engineering marks the 50th anniversary of the first laser operation by Theodore Maiman, and it presents a snapshot of several subjects related to lasers and their applications.

Although the laser field is an interdisciplinary one, we have organized the 13 contributed papers of this special section into three main groups:

1. Review papers

2. Physics, modeling, and nonlinear phenomena

3. Applications.

The first group includes manuscripts that provide a general account as to the history of the laser and its various applications. A second paper in this group reviews the status of high-power lasers in the battlefield. The second group of papers deals with physical and dynamic processes in lasers and in laser materials, with wavelength-switchable fiber lasers, resonators and laser optics, nonlinear phenomena, and modeling of Raman fiber lasers. The third group presents applications utilizing lasers such as intracavity absorption spectroscopy, optical pumping of helium atoms, and polarization and saturation effects in dye lasers. The review manuscript written by Jeff Hecht will also appear jointly in a special issue of OSA's Applied Optics, edited by Robert Boyd. This OSA special issue contains other review papers pertaining to laser history and novel applications not covered in this Optical Engineering special section. We believe that the SPIE Optical Engineering special section combined with OSA's Applied Optics issue will provide the audience with a more general overview on the subject. We are aware that these papers represent only a small fraction of the issues related to lasers and their applications. The field of lasers and laser applications is indeed very broad, and as editors, we realize that it is impossible to cover the topic completely in one special section; thus, we have opted to strive to highlight several significant points. We do hope that the audience for this special section finds it to be a valuable and worthwhile collection of manuscripts.

Finally, we would like to thank all of the authors for their insightful and timely publications. Special thanks are also extended for the efforts of the reviewers all over the world for their efforts to maintain the scientific merit of these manuscripts and therefore enhance the quality of this special section. Last but not least, we would like to thank Dr. Ronald G. Driggers, editor of Optical Engineering, Anne Munger, Karolyn Labes, and other SPIE staff members from the editorial office of Optical Engineering for their endless efforts in organizing and coordinating the process, which has resulted in such a useful special section.

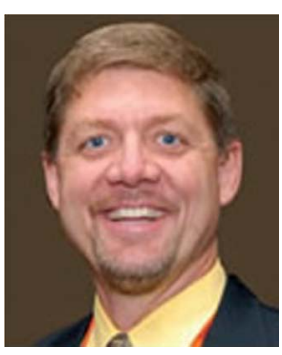

Gregory J. Quarles joined B.E. Meyers \& Co. in Redmond, Washington as the president and chief operations officer in May 2010. He was previously employed as the director of corporate research, development, and technology for II-VI Incorporated for nearly 18 years, where he developed new solid-state laser materials and laser devices for medical, military, and industrial applications. He earned a dual BS in math and physics, and MS and PhD degrees in physics from Oklahoma State University. Greg is an associate editor for both Optical Engineering and IEEE Journal of Quantum Electronics. $\mathrm{He}$ is also a Fellow of both SPIE and OSA, and has served as conference and symposia chair for various laser-related conferences for the Optical Society of America, the IEEE Lasers and Electro-Optics Society, and SPIE. During his previous 26 years of lasers and materials research, he has been awarded five patents related to solid-state lasers and has published over 100 peerreviewed publications and three books and chapters. 


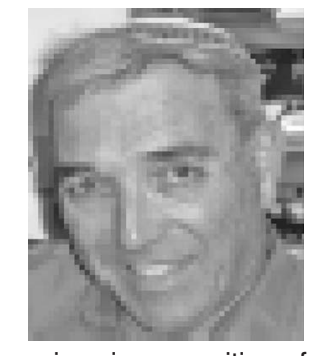

Yehoshua Kalisky is a senior scientist at Nuclear Research Center Negev. He graduated from the Hebrew University of Jerusalem in chemistry and physics, followed by a postdoctoral fellowship at Xerox Corp. In recent years he was instrumental in the design of solid-state laser systems, and the development of novel types of passively $\mathrm{Q}$-switched, diode-pumped solid-state lasers and relevant technologies for industrial applications. He was awarded several prizes in recognition of his achievements including a prize for excel- lent work (1974, 1979), a Medal of Excellence by the President of Lyon University (2002), a Prize for Excellent Optical System Design (2002), and a National Prize (2007). Dr. Kalisky is an SPIE Fellow (2007), and he is the author of the book The Physics and Engineering of Solid State Lasers. He is also the author and coauthor of over 240 scientific publications, 5 international patents, and numerous conference invited presentations. 